\title{
Strichartz inequalities for the wave equation with the full Laplacian on the Heisenberg group
}

\author{
Giulia FURIOLI \\ Dipartimento di Ingegneria Gestionale e dell'Informazione, Università di Bergamo, \\ Viale Marconi 5, I-24044 Dalmine (BG), Italy \\ E-mail: gfurioli@unibg.it, \\ Camillo MELZI \\ Dipartimento di Scienze Chimiche, Fisiche e Matematiche, Università dell'Insubria, \\ Via Valleggio 11, I-22100 Como, Italy \\ E-mail: melzi@uninsubria.it \\ and Alessandro VENERUSO \\ Dipartimento di Matematica, Università di Genova, \\ Via Dodecaneso 35, I-16146 Genova, Italy \\ E-mail: veneruso@dima.unige.it
}

\begin{abstract}
We prove dispersive and Strichartz inequalities for the solution of the wave equation related to the full Laplacian on the Heisenberg group, by means of Besov spaces defined by a Littlewood-Paley decomposition related to the spectral resolution of the full Laplacian. This requires a careful analysis due also to the non-homogeneous nature of the full Laplacian. This result has to be compared to a previous one by Bahouri, Gérard and Xu concerning the solution of the wave equation related to the Kohn-Laplacian.
\end{abstract}

Keywords: Strichartz inequalities, wave equation, full Laplacian, Heisenberg group. 2000 Mathematics Subject Classification: 22E25, 35B65. 


\section{Introduction}

The aim of this paper is to study Strichartz inequalities for the solution of the following Cauchy problem for the wave equation on the Heisenberg group $\mathbb{H}_{n}$ of topological dimension $2 n+1$ and homogeneous dimension $N=2 n+2$ :

$$
\left\{\begin{array}{l}
\partial_{t}^{2} u+\mathcal{L} u=f \in L^{1}\left((0, T), L^{2}\left(\mathbb{H}_{n}\right)\right) \\
u(0)=u_{0} \in \dot{B}_{2}^{1,2}(\mathcal{L}) \\
\partial_{t} u(0)=u_{1} \in L^{2}\left(\mathbb{H}_{n}\right)
\end{array}\right.
$$

where $\mathcal{L}$ is the full Laplacian on $\mathbb{H}_{n}$ (to be defined in Section 2) and the Besov spaces $\dot{B}_{r}^{\rho, q}(\mathcal{L})$ are defined by a Littlewood-Paley decomposition related to the spectral resolution of the full Laplacian (see Section 3). In BGX], Bahouri, Gérard and Xu studied the analogous Cauchy problem with the Kohn-Laplacian $\Delta$ instead of the full Laplacian $\mathcal{L}$, using the Besov spaces $\dot{B}_{r}^{\rho, q}(\Delta)$ which contain $\dot{B}_{r}^{\rho, q}(\mathcal{L})$ for $\rho>0$ (see Proposition [7). In [FV], the first and last authors studied the corresponding Cauchy problem for the Schrödinger equation where they introduced the full Laplacian instead of the Kohn-Laplacian, but still they used the Besov spaces $\dot{B}_{r}^{\rho, q}(\Delta)$.

Let us begin by recalling the structure of the solution of the Cauchy problem (10). It is well-known that the solution of (1I) can be written as $u=v+w$ where $v$ is the solution of (II) with $f=0$ and $w$ is the solution of (10) with $u_{0}=u_{1}=0$. More precisely,

$$
v(t)=\cos (t \sqrt{\mathcal{L}}) u_{0}+\frac{\sin (t \sqrt{\mathcal{L}})}{\sqrt{\mathcal{L}}} u_{1}
$$

and

$$
w(t)=\int_{0}^{t} \frac{\sin ((t-\sigma) \sqrt{\mathcal{L}})}{\sqrt{\mathcal{L}}} f(\sigma) d \sigma .
$$

We can now state the main results of this paper. As always when dealing with Strichartz inequalities, we prove first the following dispersive inequality on $v$.

\section{Proposition 1}

Let $\rho \in\left[N-\frac{3}{2}, N-\frac{1}{2}\right]$ and $u_{0} \in \dot{B}_{1}^{\rho, 1}(\mathcal{L}), u_{1} \in \dot{B}_{1}^{\rho-1,1}(\mathcal{L})$. Then, there exists a constant $C>0$, which does not depend on $u_{0}, u_{1}$, such that

$$
\|v(t)\|_{L^{\infty}\left(\mathbb{H}_{n}\right)} \leq C|t|^{-\frac{1}{2}}\left(\left\|u_{0}\right\|_{\dot{B}_{1}^{\rho, 1}(\mathcal{L})}+\left\|u_{1}\right\|_{\dot{B}_{1}^{\rho-1,1}(\mathcal{L})}\right), \quad t \in \mathbb{R}^{*}
$$

Let us remark the main difference between Proposition 1 and BGX, Théorème 1.2]: in the

hypotheses of the latter theorem, they obtain only the index $\rho=N-\frac{1}{2}$, which in that case is sharp because of the homogeneity property of the Kohn-Laplacian $\Delta$.

For every interval $I \subset \mathbb{R}$ we will denote by $L_{I}^{p}(X)$ the space $L^{p}(I, X)$. The Strichartz inequalities we have obtained are the following.

\section{Theorem 2}

Let $r_{1}, r_{2} \in[2, \infty]$. Let $\rho_{1}, \rho_{2} \in \mathbb{R}$ and $p_{1}, p_{2} \in[1, \infty]$ such that: 
a) $\frac{2}{p_{i}}=\frac{1}{2}-\frac{1}{r_{i}}$ for $i=1,2$;

b) $-\left(N-\frac{1}{2}\right)\left(\frac{1}{2}-\frac{1}{r_{1}}\right)+1 \leq \rho_{1} \leq-\left(N-\frac{3}{2}\right)\left(\frac{1}{2}-\frac{1}{r_{1}}\right)+1$;

c) $-\left(N-\frac{1}{2}\right)\left(\frac{1}{2}-\frac{1}{r_{2}}\right) \leq \rho_{2} \leq-\left(N-\frac{3}{2}\right)\left(\frac{1}{2}-\frac{1}{r_{2}}\right)$.

Let $r_{i}^{\prime}, p_{i}^{\prime}$ such that $\frac{1}{r_{i}^{\prime}}+\frac{1}{r_{i}}=1$ and $\frac{1}{p_{i}^{\prime}}+\frac{1}{p_{i}}=1$ for $i=1,2$. Then for every interval $I$ which contains 0 the following estimates are satisfied:

$$
\begin{aligned}
& \|v\|_{L_{\mathbb{R}}^{p_{1}}\left(\dot{B}_{r_{1}}^{\rho_{1}, 2}(\mathcal{L})\right)}+\left\|\partial_{t} v\right\|_{L_{\mathbb{R}}^{p_{1}}\left(\dot{B}_{r_{1}}^{\rho_{1}-1,2}(\mathcal{L})\right)} \leq C\left(\left\|u_{0}\right\|_{\dot{B}_{2}^{1,2}(\mathcal{L})}+\left\|u_{1}\right\|_{L^{2}\left(\mathbb{H}_{n}\right)}\right) \\
& \|w\|_{L_{I}^{p_{1}}\left(\dot{B}_{r_{1}}^{\rho_{1}, 2}(\mathcal{L})\right)}+\left\|\partial_{t} w\right\|_{L_{I}^{p_{1}}\left(\dot{B}_{r_{1}}^{\rho_{1}-1,2}(\mathcal{L})\right)} \leq C\|f\|_{L_{I}^{p_{2}^{\prime}\left(\dot{B}_{r_{2}^{\prime}}^{-\rho_{2}, 2}(\mathcal{L})\right)}}
\end{aligned}
$$

where the constant $C>0$ depends neither on $u_{0}, u_{1}, f$ nor on the interval $I$.

So, we can deduce from Theorem 2 the following result, which we compare to the analogous result by Bahouri, Gérard and $\mathrm{Xu}$.

\section{Corollary 3}

Let $u$ be the solution of the Cauchy problem (10). If $p$ and $r$ satisfy $0 \leq \frac{2}{p} \leq \frac{1}{2}-\frac{1}{r}$ and $(N-1)\left(\frac{1}{2}-\frac{1}{r}\right)-1 \leq \frac{1}{p} \leq N\left(\frac{1}{2}-\frac{1}{r}\right)-1$, then there exists a constant $C>0$, which does not depend on $u_{0}, u_{1}, f$, such that for every interval $I$ which contains 0 the following estimate is satisfied:

$$
\|u\|_{L_{I}^{p}\left(L^{r}\left(\mathbb{H}_{n}\right)\right)} \leq C\left(\left\|u_{0}\right\|_{\dot{B}_{2}^{1,2}(\mathcal{L})}+\left\|u_{1}\right\|_{L^{2}\left(\mathbb{H}_{n}\right)}+\|f\|_{L_{I}^{1}\left(L^{2}\left(\mathbb{H}_{n}\right)\right)}\right) .
$$

In [BGX, Théorème 1.1], the solution of the wave equation with the Kohn-Laplacian was proved to belong to $L_{I}^{p}\left(L^{r}\left(\mathbb{H}_{n}\right)\right)$ only for $p$ and $r$ satisfying $2 N-1 \leq p \leq \infty$ and $\frac{1}{p}=N\left(\frac{1}{2}-\frac{1}{r}\right)-1$ which is a subset of the range of values of $p$ and $r$ we have found (since it is equivalent to $0 \leq \frac{2}{p} \leq \frac{1}{2}-\frac{1}{r}$ and $\left.\frac{1}{p}=N\left(\frac{1}{2}-\frac{1}{r}\right)-1\right)$. The set of the admissible values $\left(\frac{1}{r}, \frac{1}{p}\right)$ found in Corollary 3 is represented in the picture, where the result by Bahouri, Gérard and $\mathrm{Xu}$ corresponds to the segment $B C$.

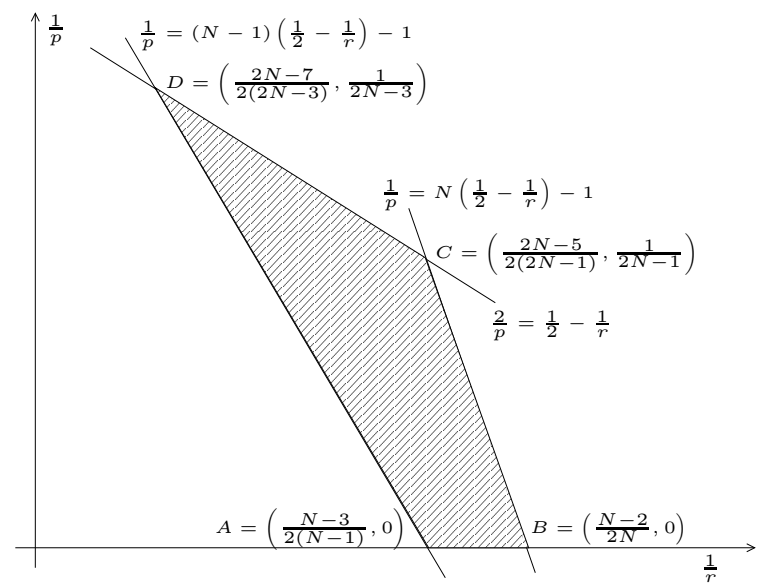

Other results on the sharpness of the dispersive inequalities and remarks about the behaviour of the operator $e^{-i t \sqrt{\mathcal{L}}}$ when analysed by the Besov spaces $\dot{B}_{r}^{\rho, q}(\Delta)$ can be found in Section [6] 


\section{Notation and preliminaries}

In this paper $\mathbb{N}$ denotes the set of nonnegative integers, $\mathbb{Z}_{+}$the set of positive integers and $\mathbb{R}_{+}$the set of positive real numbers. For $p \in[1, \infty]$ we denote by $p^{\prime}$ the conjugate index of $p$, such that $\frac{1}{p}+\frac{1}{p^{\prime}}=1$. We will denote by $C$ any positive constant, depending only on the group, which will not be necessarily the same at each occurrence.

In this section we recall some basic facts about harmonic analysis on the Heisenberg group. For the proofs and further information, see e.g. [BJRW], F], Ge, [N].

The Heisenberg group $\mathbb{H}_{n}, n \in \mathbb{Z}_{+}$, is the nilpotent Lie group whose underlying manifold is $\mathbb{R}^{n} \times \mathbb{R}^{n} \times \mathbb{R}$, with the following multiplication law:

$$
(x, y, s)\left(x^{\prime}, y^{\prime}, s^{\prime}\right)=\left(x+x^{\prime}, y+y^{\prime}, s+s^{\prime}+2\left(y \cdot x^{\prime}-x \cdot y^{\prime}\right)\right), \quad x, x^{\prime}, y, y^{\prime} \in \mathbb{R}^{n}, s, s^{\prime} \in \mathbb{R} .
$$

The Lie algebra of $\mathbb{H}_{n}$ is generated by the left-invariant vector fields $X_{1}, \ldots, X_{n}, Y_{1}, \ldots, Y_{n}, S$, where

$$
X_{j}=\frac{\partial}{\partial x_{j}}+2 y_{j} \frac{\partial}{\partial s}, \quad Y_{j}=\frac{\partial}{\partial y_{j}}-2 x_{j} \frac{\partial}{\partial s}, \quad S=\frac{\partial}{\partial s} .
$$

We indicate an element $g=(x, y, s) \in \mathbb{H}_{n}$ as $g=(z, s)$, where $z=x+i y \in \mathbb{C}^{n}$. The family of dilations $\left\{\delta_{r}: r>0\right\}$ given by

$$
\delta_{r}(z, s)=\left(r z, r^{2} s\right)
$$

makes $\mathbb{H}_{n}$ a stratified group of homogeneous dimension $N=2 n+2$. The Kohn-Laplacian

$$
\Delta=-\sum_{j=1}^{n}\left(X_{j}^{2}+Y_{j}^{2}\right)
$$

satisfies the homogeneity property $\Delta\left(f \circ \delta_{r}\right)=r^{2}\left(\Delta f \circ \delta_{r}\right), r>0$, while the full Laplacian

$$
\mathcal{L}=\Delta-S^{2}
$$

is not invariant with respect to the dilation structure of $\mathbb{H}_{n}$.

The bi-invariant Haar measure $d g$ on $\mathbb{H}_{n}$ coincides with the Lebesgue measure on $\mathbb{R}^{2 n+1}$. The convolution of two functions $f_{1}$ and $f_{2}$ on $G$, defined by

$$
f_{1} * f_{2}(g)=\int_{\mathbb{H}_{n}} f_{1}\left(g g^{\prime-1}\right) f_{2}\left(g^{\prime}\right) d g^{\prime}, \quad g \in \mathbb{H}_{n},
$$

satisfies the Young's inequality (where $1+\frac{1}{r}=\frac{1}{p}+\frac{1}{q}$ )

$$
\left\|f_{1} * f_{2}\right\|_{L^{r}\left(\mathbb{H}_{n}\right)} \leq\left\|f_{1}\right\|_{L^{p}\left(\mathbb{H}_{n}\right)}\left\|f_{2}\right\|_{L^{q}\left(\mathbb{H}_{n}\right)} .
$$

The convolution of $\varphi \in \mathcal{S}\left(\mathbb{H}_{n}\right)$ and $u \in \mathcal{S}^{\prime}\left(\mathbb{H}_{n}\right)$, where $\mathcal{S}\left(\mathbb{H}_{n}\right)$ is the Schwartz space and $\mathcal{S}^{\prime}\left(\mathbb{H}_{n}\right)$ is the space of tempered distributions, is defined as usual (see e.g. [V]). We say that a function $f$ on $\mathbb{H}_{n}$ is radial if the value of $f(z, s)$ depends only on $|z|$ and $s$. We denote by $\mathcal{S}_{\text {rad }}\left(\mathbb{H}_{n}\right)$ and by $L_{\text {rad }}^{p}\left(\mathbb{H}_{n}\right), 1 \leq p \leq \infty$, the spaces of radial functions in $\mathcal{S}\left(\mathbb{H}_{n}\right)$ and in $L^{p}\left(\mathbb{H}_{n}\right)$, respectively. The space $L_{\text {rad }}^{1}\left(\mathbb{H}_{n}\right)$ is a commutative, closed $*$-subalgebra of $L^{1}\left(\mathbb{H}_{n}\right)$. The Gelfand spectrum 
$\Sigma$ of $L_{\text {rad }}^{1}\left(\mathbb{H}_{n}\right)$ can be identified, as a measure space, with the space $\mathbb{N} \times \mathbb{R}$ equipped with the Godement-Plancherel measure $\mu$ defined by

$$
\int_{\Sigma} F(\psi) d \mu(\psi)=\frac{2^{n-1}}{\pi^{n+1}} \sum_{m=0}^{+\infty}\left(\begin{array}{c}
m+n-1 \\
m
\end{array}\right) \int_{\mathbb{R}} F(m, \lambda)|\lambda|^{n} d \lambda .
$$

The spherical Fourier transform of a function $f \in L_{\text {rad }}^{1}\left(\mathbb{H}_{n}\right)$ is given by

$$
\hat{f}(m, \lambda)=\int_{\mathbb{H}_{n}} f(g) \omega_{m, \lambda}(g) d g, \quad m \in \mathbb{N}, \lambda \in \mathbb{R},
$$

with

$$
\omega_{m, \lambda}(z, s)=\left(\begin{array}{c}
m+n-1 \\
m
\end{array}\right)^{-1} e^{i \lambda s} e^{-|\lambda||z|^{2}} L_{m}^{(n-1)}\left(2|\lambda||z|^{2}\right),
$$

where $L_{m}^{(\alpha)}$ is the Laguerre polynomial of type $\alpha \in \mathbb{N}$ and degree $m \in \mathbb{N}$, defined by

$$
L_{m}^{(\alpha)}(\tau)=\sum_{k=0}^{m} \frac{(-1)^{k}}{k !}\left(\begin{array}{c}
m+\alpha \\
k+\alpha
\end{array}\right) \tau^{k}, \quad \tau \in \mathbb{R}
$$

We have $\widehat{f_{1} * f_{2}}=\widehat{f}_{1} \widehat{f}_{2}$ for any $f_{1}, f_{2} \in L_{\text {rad }}^{1}\left(\mathbb{H}_{n}\right)$. Since $\left\|\omega_{m, \lambda}\right\|_{L^{\infty}\left(\mathbb{H}_{n}\right)}=1$ the spherical Fourier transform is bounded from $L_{\text {rad }}^{1}\left(\mathbb{H}_{n}\right)$ to $L^{\infty}(\Sigma)$. Moreover, by the GodementPlancherel theory, it extends uniquely to a unitary operator $\mathcal{G}: L_{\text {rad }}^{2}\left(\mathbb{H}_{n}\right) \longrightarrow L^{2}(\Sigma)$. We still write $\hat{f}$ instead of $\mathcal{G} f$. If $f \in L_{\text {rad }}^{2}\left(\mathbb{H}_{n}\right)$ and $\hat{f} \in L^{1}(\Sigma)$, the following inversion formula holds:

$$
f(g)=\frac{2^{n-1}}{\pi^{n+1}} \sum_{m=0}^{+\infty}\left(\begin{array}{c}
m+n-1 \\
m
\end{array}\right) \int_{\mathbb{R}} \hat{f}(m, \lambda) \omega_{m,-\lambda}(g)|\lambda|^{n} d \lambda, \quad g \in \mathbb{H}_{n} .
$$

The space $\mathcal{G}\left(\mathcal{S}_{\text {rad }}\left(\mathbb{H}_{n}\right)\right)$ has been described in BJR. For our purposes, it is sufficient to remark that $\mathcal{G}\left(\mathcal{S}_{\text {rad }}\left(\mathbb{H}_{n}\right)\right) \subset L^{1}(\Sigma)$. Moreover, if $f \in \mathcal{S}_{\text {rad }}\left(\mathbb{H}_{n}\right)$ the functions $\Delta f$ and $\mathcal{L} f$ are in $\mathcal{S}_{\text {rad }}\left(\mathbb{H}_{n}\right)$ and their spherical Fourier transforms are given by:

$$
\begin{aligned}
\widehat{\Delta f}(m, \lambda) & =4(2 m+n)|\lambda| \hat{f}(m, \lambda), \\
\widehat{\mathcal{L} f}(m, \lambda) & =\left(4(2 m+n)|\lambda|+\lambda^{2}\right) \hat{f}(m, \lambda) .
\end{aligned}
$$

Both $\Delta$ and $\mathcal{L}$ are positive self-adjoint operators densely defined on $L^{2}\left(\mathbb{H}_{n}\right)$. So, by the spectral theorem, for any bounded Borel function $h$ on $[0,+\infty)$ the operators $h(\Delta)$ and $h(\mathcal{L})$ are bounded on $L^{2}\left(\mathbb{H}_{n}\right)$. Since the point 0 may be neglected in the spectral resolution (see $[\mathrm{A}$, C] $)$, we consider that the function $h$ is defined on $\mathbb{R}_{+}$. If $f \in L_{\text {rad }}^{2}\left(\mathbb{H}_{n}\right)$ the functions $h(\Delta) f$ and $h(\mathcal{L}) f$ are in $L_{\text {rad }}^{2}\left(\mathbb{H}_{n}\right)$ and their spherical Fourier transforms, by (5) and (6), are given by:

$$
\begin{aligned}
\widehat{h(\Delta) f}(m, \lambda) & =h(4(2 m+n)|\lambda|) \hat{f}(m, \lambda), \\
\widehat{h(\mathcal{L}) f}(m, \lambda) & =h\left(4(2 m+n)|\lambda|+\lambda^{2}\right) \hat{f}(m, \lambda) .
\end{aligned}
$$

If $f \in \mathcal{S}_{\text {rad }}\left(\mathbb{H}_{n}\right)$ then, by the previous remarks, the functions $h(\Delta) f$ and $h(\mathcal{L}) f$ can be recovered from their spherical Fourier transforms by means of the inversion formula (44). 
The operators $h(\Delta)$ and $h(\mathcal{L})$ commute with left translations. So by the Schwartz' kernel theorem, which is valid also on $\mathbb{H}_{n}$ (see [KVW] Theorem 3.2]), they admit kernels in $\mathcal{S}^{\prime}\left(\mathbb{H}_{n}\right)$, which we call $H_{\Delta}$ and $H_{\mathcal{L}}$ respectively, satisfying $h(\Delta) f=f * H_{\Delta}$ and $h(\mathcal{L}) f=f * H_{\mathcal{L}}$ for any $f \in \mathcal{S}\left(\mathbb{H}_{n}\right)$. If $h$ is the restriction on $\mathbb{R}_{+}$of a function in $\mathcal{S}(\mathbb{R})$, then $H_{\Delta}$ and $H_{\mathcal{L}}$ are in $\mathcal{S}_{\text {rad }}\left(\mathbb{H}_{n}\right)$ (see [FMV, Corollary 7]; see also [Hu, [M] for $H_{\Delta}$, V] for $H_{\mathcal{L}}$ ) and their spherical Fourier transforms, by (7) and (8), are given by:

$$
\begin{aligned}
\widehat{H_{\Delta}}(m, \lambda) & =h(4(2 m+n)|\lambda|), \\
\widehat{H_{\mathcal{L}}}(m, \lambda) & =h\left(4(2 m+n)|\lambda|+\lambda^{2}\right) .
\end{aligned}
$$

\section{Littlewood-Paley decompositions and Besov spaces}

Let $R$ be a non-negative function in $C^{\infty}(\mathbb{R})$ such that $\operatorname{supp} R \subset\left[\frac{1}{4}, 4\right]$ and

$$
\sum_{j \in \mathbb{Z}} R\left(2^{-2 j} \tau\right)=1, \quad \tau>0
$$

For any $j \in \mathbb{Z}$ we denote by $\varphi_{j}$ and $\psi_{j}$ the kernels of the operators $R\left(2^{-2 j} \Delta\right)$ and $R\left(2^{-2 j} \mathcal{L}\right)$, respectively. The remarks at the end of Section 2 guarantee that $\varphi_{j}, \psi_{j} \in \mathcal{S}_{\text {rad }}\left(\mathbb{H}_{n}\right)$ and

$$
\begin{aligned}
& \widehat{\varphi_{j}}(m, \lambda)=R\left(2^{2-2 j}(2 m+n)|\lambda|\right), \\
& \widehat{\psi_{j}}(m, \lambda)=R\left(2^{-2 j}\left(4(2 m+n)|\lambda|+\lambda^{2}\right)\right) .
\end{aligned}
$$

If $j, k \in \mathbb{Z}$ with $|j-k| \geq 2$, then $\varphi_{j} * \varphi_{k}=\psi_{j} * \psi_{k}=0$. Moreover we have the following

\section{Lemma 4}

For any $j \in \mathbb{Z}$ the sets $U_{j}=\left\{k \in \mathbb{Z}: \varphi_{j} * \psi_{k} \neq 0\right\}$ and $V_{j}=\left\{k \in \mathbb{Z}: \psi_{j} * \varphi_{k} \neq 0\right\}$ are finite and $\min U_{j} \geq j-2, \max V_{j} \leq j+2$.

Proof: Fix $j \in \mathbb{Z}$ and $k \in U_{j}$. By (9) and (10) there exist $m \in \mathbb{N}, \lambda \in \mathbb{R}$ such that

$$
R\left(2^{2-2 j}(2 m+n)|\lambda|\right) R\left(2^{-2 k}\left(4(2 m+n)|\lambda|+\lambda^{2}\right)\right) \neq 0 .
$$

Put $\xi=4(2 m+n)|\lambda|$ and $\eta=\lambda^{2}$. The pair $(\xi, \eta)$ satisfies the following system of inequalities:

$$
\left\{\begin{array}{l}
\frac{1}{4} \leq 2^{-2 j} \xi \leq 4 \\
\frac{1}{4} \leq 2^{-2 k}(\xi+\eta) \leq 4 \\
0 \leq \eta \leq \frac{\xi^{2}}{16 n^{2}} .
\end{array}\right.
$$

On the other hand, it is easy to check that the system (11) admits solutions only if

$$
2^{2 j-4} \leq 2^{2 k} \leq \frac{2^{4 j+2}}{n^{2}}+2^{2 j+4}
$$

These conditions give the conclusion not only for $U_{j}$, but also for $V_{j}$ : for the latter one it is sufficient to interchange the roles of $j$ and $k$, noting that $k \in V_{j}$ if and only if $j \in U_{k}$. 
A direct application of the inversion formula (4) gives

$$
\varphi_{j}(z, s)=2^{N j} \varphi_{0}\left(2^{j} z, 2^{2 j} s\right), \quad j \in \mathbb{Z},(z, s) \in \mathbb{H}_{n} .
$$

So

$$
\left\|\varphi_{j}\right\|_{L^{1}\left(\mathbb{H}_{n}\right)}=\left\|\varphi_{0}\right\|_{L^{1}\left(\mathbb{H}_{n}\right)}, \quad j \in \mathbb{Z} .
$$

On the other hand, despite the lack of homogeneity, by [FMV, Proposition 6] there exists $C>0$ such that

$$
\left\|\psi_{j}\right\|_{L^{1}\left(\mathbb{H}_{n}\right)} \leq C, \quad j \in \mathbb{Z}
$$

In this section, in order to carry on some results which are valid for both operators $\Delta$ and $\mathcal{L}$, we use the notation $L$ to denote either $\Delta$ or $\mathcal{L}$. For any $u \in \mathcal{S}^{\prime}\left(\mathbb{H}_{n}\right)$, if $L=\Delta$ we set $\Delta_{j} u=u * \varphi_{j}$, if $L=\mathcal{L}$ we set $\Delta_{j} u=u * \psi_{j}$. By standard arguments (see e.g. [FMV], Proposition 9]) we can deduce from (13) and (14) that

$$
\left\|L^{\frac{\sigma}{2}} \Delta_{j} u\right\|_{L^{p}\left(\mathbb{H}_{n}\right)} \leq C 2^{j \sigma}\left\|\Delta_{j} u\right\|_{L^{p}\left(\mathbb{H}_{n}\right)}, \quad \sigma \in \mathbb{R}, j \in \mathbb{Z}, 1 \leq p \leq \infty, u \in \mathcal{S}^{\prime}\left(\mathbb{H}_{n}\right),
$$

where both sides of (15) are allowed to be infinite.

By the spectral theorem, for any $f \in L^{2}\left(\mathbb{H}_{n}\right)$ the following homogeneous Littlewood-Paley decomposition holds:

$$
f=\sum_{j \in \mathbb{Z}} \Delta_{j} f \quad \text { in } L^{2}\left(\mathbb{H}_{n}\right)
$$

So

$$
\|f\|_{L^{\infty}\left(\mathbb{H}_{n}\right)} \leq \sum_{j \in \mathbb{Z}}\left\|\Delta_{j} f\right\|_{L^{\infty}\left(\mathbb{H}_{n}\right)}, \quad f \in L^{2}\left(\mathbb{H}_{n}\right),
$$

where both sides of (17) are allowed to be infinite.

The methods of $[\mathrm{St}$ ], together with any multiplier theorem for $L$ (see $\underline{\mathrm{A}}$; see also $\mathrm{He}$, [MS] for $L=\Delta$, MRS1, MRS2 for $L=\mathcal{L}$ ), yield the following Littlewood-Paley theorem:

\section{Proposition 5}

Let $1<p<\infty$ and $u \in \mathcal{S}^{\prime}\left(\mathbb{H}_{n}\right)$. The following facts are equivalent:

(i) $u \in L^{p}\left(\mathbb{H}_{n}\right)$;

(ii) $u=\sum_{j \in \mathbb{Z}} \Delta_{j} u$ in $\mathcal{S}^{\prime}\left(\mathbb{H}_{n}\right)$ and $\left(\sum_{j \in \mathbb{Z}}\left|\Delta_{j} u\right|^{2}\right)^{\frac{1}{2}} \in L^{p}\left(\mathbb{H}_{n}\right)$.

Moreover, if $u \in L^{p}\left(\mathbb{H}_{n}\right)$ then

$$
\|u\|_{L^{p}\left(\mathbb{H}_{n}\right)} \sim\left\|\left(\sum_{j \in \mathbb{Z}}\left|\Delta_{j} u\right|^{2}\right)^{\frac{1}{2}}\right\|_{L^{p}\left(\mathbb{H}_{n}\right)} .
$$

Remark: For $L=\Delta$ Proposition [5 has been proved also in [BGX, Proposition 2.3] using the homogeneity property (12).

Let $q, r \in[1, \infty]$ and $\rho \in \mathbb{R}$; the homogeneous Besov space $\dot{B}_{r}^{\rho, q}(L)$ associated to the operator $L$ is defined as follows:

$$
\dot{B}_{r}^{\rho, q}(L)=\left\{u \in \mathcal{S}^{\prime}\left(\mathbb{H}_{n}\right): u=\sum_{j \in \mathbb{Z}} \Delta_{j} u \text { in } \mathcal{S}^{\prime}\left(\mathbb{H}_{n}\right) \text { and }\left\{2^{j \rho}\left\|\Delta_{j} u\right\|_{L^{r}\left(\mathbb{H}_{n}\right)}\right\}_{j \in \mathbb{Z}} \in l^{q}(\mathbb{Z})\right\} .
$$

We collect in the following proposition all the properties we need about the spaces $\dot{B}_{r}^{\rho, q}(L)$. 


\section{Proposition 6}

Let $q, r \in[1, \infty]$ and $\rho<\frac{N}{r}$.

(i) The space $\dot{B}_{r}^{\rho, q}(L)$ is a Banach space endowed with the norm

$$
\|u\|_{\dot{B}_{r}^{\rho, q}(L)}=\left\|\left\{2^{j \rho}\left\|\Delta_{j} u\right\|_{L^{r}\left(\mathbb{H}_{n}\right)}\right\}_{j \in \mathbb{Z}}\right\|_{l^{q}(\mathbb{Z})}
$$

(ii) the definition of $\dot{B}_{r}^{\rho, q}(L)$ does not depend on the choice of the function $R$ in the LittlewoodPaley decomposition;

(iii) for any $u \in \mathcal{S}^{\prime}\left(\mathbb{H}_{n}\right)$ and $\sigma>0$ we have that $u \in \dot{B}_{r}^{\rho, q}(L)$ if and only if $L^{\frac{\sigma}{2}} u \in \dot{B}_{r}^{\rho-\sigma, q}(L)$, with

$$
\|u\|_{\dot{B}_{r}^{\rho, q}(L)} \sim\left\|L^{\frac{\sigma}{2}} u\right\|_{\dot{B}_{r}^{\rho-\sigma, q}(L)}
$$

(iv) the inclusion $\dot{B}_{r}^{\rho, q}(L) \subset \mathcal{S}^{\prime}\left(\mathbb{H}_{n}\right)$ is continuous;

(v) if $-\frac{N}{r^{\prime}}<\rho<\frac{N}{r}$ then $\mathcal{S}\left(\mathbb{H}_{n}\right) \subset \dot{B}_{r}^{\rho, q}(L)$ with continuous inclusion;

(vi) if $q, r \in[1, \infty)$ and $-\frac{N}{r^{\prime}}<\rho<\frac{N}{r}$, then $\mathcal{S}\left(\mathbb{H}_{n}\right)$ is dense in $\dot{B}_{r}^{\rho, q}(L)$;

(vii) if $q, r \in[1, \infty)$ and $-\frac{N}{r^{\prime}}<\rho<\frac{N}{r}$, the dual space of $\dot{B}_{r}^{\rho, q}(L)$ is $\dot{B}_{r^{\prime}}^{-\rho, q^{\prime}}(L)$;

(viii) for all $q \in[1, \infty]$ and $\alpha \in[N-1, N]$ we have the continuos inclusions

$$
\begin{aligned}
& \dot{B}_{r_{1}}^{\rho_{1}, q}(\mathcal{L}) \subset \dot{B}_{r_{2}}^{\rho_{2}, q}(\mathcal{L}), \quad \frac{1}{r_{1}}-\frac{\rho_{1}}{\alpha}=\frac{1}{r_{2}}-\frac{\rho_{2}}{\alpha}, \rho_{1} \geq \rho_{2} ; \\
& \dot{B}_{r_{1}}^{\rho_{1}, q}(\Delta) \subset \dot{B}_{r_{2}}^{\rho_{2}, q}(\Delta), \quad \frac{1}{r_{1}}-\frac{\rho_{1}}{N}=\frac{1}{r_{2}}-\frac{\rho_{2}}{N}, \rho_{1} \geq \rho_{2}
\end{aligned}
$$

(ix) for all $r \in[2, \infty)$ we have the continuos inclusion $\dot{B}_{r}^{0,2}(L) \subset L^{r}\left(\mathbb{H}_{n}\right)$;

(x) $\dot{B}_{2}^{0,2}(L)=L^{2}\left(\mathbb{H}_{n}\right)$ with equivalent norms;

(xi) for all $\vartheta, \rho_{1}, \rho_{2}, q_{1}, q_{2}, r_{1}, r_{2}$ satisfying $\vartheta \in[0,1], q_{i}, r_{i} \in(1, \infty), \rho_{i}<\frac{N}{r_{i}}$, we have

$$
\left[\dot{B}_{r_{1}}^{\rho_{1}, q_{1}}(L), \dot{B}_{r_{2}}^{\rho_{2}, q_{2}}(L)\right]_{\vartheta}=\dot{B}_{r}^{\rho, q}(L)
$$

$$
\text { with } \rho=(1-\vartheta) \rho_{1}+\vartheta \rho_{2}, \frac{1}{q}=\frac{1-\vartheta}{q_{1}}+\frac{\vartheta}{q_{2}} \text { and } \frac{1}{r}=\frac{1-\vartheta}{r_{1}}+\frac{\vartheta}{r_{2}} \text {. }
$$

We omit the proof of Proposition [ 6 In fact, all the statements of the proposition are wellknown for the spaces $\dot{B}_{r}^{\rho, q}(\Delta)$ (see [BG], [BGX], [FV]) and the proofs for the spaces $\dot{B}_{r}^{\rho, q}(\mathcal{L})$ are analogous: the only properties really needed are estimates (14) and (15), Proposition 5 and the fact that the kernel of $h(\mathcal{L})$ is in $\mathcal{S}\left(\mathbb{H}_{n}\right)$ if $h \in \mathcal{S}(\mathbb{R})$ (see Section 2). Once we have these properties, we can prove Proposition [6] by the methods in $[\mathbf{P}]$, which do not involve any homogeneity property. More generally, we could define homogeneous Besov spaces and prove, with the same methods, an analogous proposition in the more general context of a nilpotent Lie group $G$ endowed with a sub-Laplacian $L=-\sum_{j=1}^{k} X_{j}^{2}$, where $X_{1}, \ldots, X_{k}$ are 
left-invariant vector fields on $G$ which satisfy the Hörmander's condition, i.e. they generate, together with their successive Lie brackets $\left[X_{i_{1}},\left[\ldots, X_{i_{\alpha}}\right] \cdots\right]$, the Lie algebra of $G$. For more details about properties of Besov spaces in this context, see [S1], S2], FMV], where nevertheless inhomogeneous Besov spaces are considered. Here we want to prove some continuous inclusions between the two kinds of homogeneous Besov spaces which we have introduced.

\section{Proposition 7}

The following continuos inclusions hold:

$$
\begin{aligned}
& \dot{B}_{r}^{\rho, q}(\mathcal{L}) \quad \subset \quad \dot{B}_{r}^{\rho, q}(\Delta), \quad 1 \leq q \leq \infty, 1 \leq r<\infty, 0<\rho<\frac{N}{r} \\
& \dot{B}_{r}^{\rho, q}(\Delta) \quad \subset \quad \dot{B}_{r}^{\rho, q}(\mathcal{L}), \quad 1 \leq q \leq \infty, 1<r \leq \infty,-\frac{N}{r^{\prime}}<\rho<0 .
\end{aligned}
$$

Proof: We only prove (18), since the proof of (19) is analogous. Fix $u \in \dot{B}_{r}^{\rho, q}(\mathcal{L})$, with $1 \leq q \leq \infty, 1 \leq r<\infty$ and $0<\rho<\frac{N}{r}$. Since $u=\sum_{j \in \mathbb{Z}} u * \psi_{j}$ in $\mathcal{S}^{\prime}\left(\mathbb{H}_{n}\right)$, by Lemma 4 we have $u * \varphi_{k}=\sum_{j \geq k-2} u * \psi_{j} * \varphi_{k}$ in $\mathcal{S}^{\prime}\left(\mathbb{H}_{n}\right)$ for any $k \in \mathbb{Z}$, and so

$$
\begin{aligned}
2^{k \rho}\left\|u * \varphi_{k}\right\|_{L^{r}\left(\mathbb{H}_{n}\right)} & \leq 2^{k \rho} \sum_{j \geq k-2}\left\|u * \psi_{j} * \varphi_{k}\right\|_{L^{r}\left(\mathbb{H}_{n}\right)} \\
& \leq C \sum_{j \geq k-2} 2^{(k-j) \rho} 2^{j \rho}\left\|u * \psi_{j}\right\|_{L^{r}\left(\mathbb{H}_{n}\right)}
\end{aligned}
$$

by (13). Therefore, by Young's inequality

$$
\left\|\left\{2^{k \rho}\left\|u * \varphi_{k}\right\|_{L^{r}\left(\mathbb{H}_{n}\right)}\right\}_{k \in \mathbb{Z}}\right\|_{l^{q}(\mathbb{Z})} \leq C\|u\|_{\dot{B}_{r}^{\rho, q}(\mathcal{L})} .
$$

We still have to prove that $u=\sum_{k \in \mathbb{Z}} u * \varphi_{k}$ in $\mathcal{S}^{\prime}\left(\mathbb{H}_{n}\right)$. By Lemma 4 for any $f \in \mathcal{S}\left(\mathbb{H}_{n}\right)$ we have:

$$
\begin{aligned}
\sum_{j \in \mathbb{Z}} \sum_{k \in \mathbb{Z}}\left|\left\langle u * \psi_{j} * \varphi_{k}, f\right\rangle\right| & =\sum_{j \in \mathbb{Z}} \sum_{h=-\infty}^{2}\left|\left\langle u * \psi_{j}, f * \varphi_{j+h}\right\rangle\right| \\
& \leq \sum_{h=-\infty}^{2} 2^{h \rho}\left(\sum_{j \in \mathbb{Z}} 2^{j \rho}\left\|u * \psi_{j}\right\|_{L^{r}\left(\mathbb{H}_{n}\right)} 2^{-(j+h) \rho}\left\|f * \varphi_{j+h}\right\|_{L^{r^{\prime}\left(\mathbb{H}_{n}\right)}}\right) \\
& \leq \sum_{h=-\infty}^{2} 2^{h \rho}\|u\|_{\dot{B}_{r}^{\rho, q}(\mathcal{L})}\|f\|_{\dot{B}_{r^{\prime}}^{-\rho, q^{\prime}}(\Delta)}<+\infty .
\end{aligned}
$$

Note that $\varphi_{k}=\sum_{j \in \mathbb{Z}} \varphi_{k} * \psi_{j}$ in $\mathcal{S}\left(\mathbb{H}_{n}\right)$ for any $k \in \mathbb{Z}$, by (16) and Lemma 4 . Therefore, since $u=\sum_{j \in \mathbb{Z}} u * \psi_{j}$ in $\mathcal{S}^{\prime}\left(\mathbb{H}_{n}\right)$, by Fubini's theorem we have

$$
\langle u, f\rangle=\sum_{j \in \mathbb{Z}} \sum_{k \in \mathbb{Z}}\left\langle u * \psi_{j} * \varphi_{k}, f\right\rangle=\sum_{k \in \mathbb{Z}}\left\langle u * \varphi_{k}, f\right\rangle, \quad f \in \mathcal{S}\left(\mathbb{H}_{n}\right) .
$$


However, with the exception of particular cases as $\rho=0, q=r=2$ (see Proposition [ 6 (x)), the spaces $\dot{B}_{r}^{\rho, q}(\Delta)$ and $\dot{B}_{r}^{\rho, q}(\mathcal{L})$ do not coincide: for example, by applying the GodementPlancherel's formula and arguing as in the proof of Lemma 4 . it is not hard to check that for $j \rightarrow+\infty$ we have

$$
\left\|\varphi_{j}\right\|_{\dot{B}_{2}^{\rho, q}(\Delta)} \sim 2^{j\left(\rho+\frac{N}{2}\right)}, \quad\left\|\varphi_{j}\right\|_{\dot{B}_{2}^{\rho, q}(\mathcal{L})} \sim 2^{j\left(2 \rho+\frac{N}{2}\right)}, \quad 1 \leq q \leq \infty, 0<\rho<\frac{N}{2} .
$$

As a further evidence, in the following we will see that the spaces $\dot{B}_{r}^{\rho, q}(\Delta)$ and $\dot{B}_{r}^{\rho, q}(\mathcal{L})$ have a very different behaviour with respect to Strichartz estimates for the solution of the Cauchy problem (11).

\section{Dispersive estimates}

We begin by proving Proposition 1 Let us introduce the tools of the method; first of all, we recall the stationary phase lemma (see e.g. [St, pages 332-334) that will be the central argument:

\section{Lemma 8}

Suppose $g, h \in C^{\infty}([a, b])$, with $g$ real-valued and $h(b)=0$. Suppose also $\left|g^{(k)}(x)\right| \geq \delta$ for any $x \in[a, b]$, with $k \in \mathbb{Z}_{+}$and $\delta>0$. If $k=1$, we also require that $g^{\prime}$ is monotonic in $[a, b]$. Then there exists a constant $C_{k}>0$, which depends only on $k$ but not on $a, b, g, h, \delta$, such that

$$
\left|\int_{a}^{b} e^{-i g(x)} h(x) d x\right| \leq C_{k} \delta^{-\frac{1}{k}} \int_{a}^{b}\left|h^{\prime}(x)\right| d x .
$$

Moreover, we will use the following properties of the Laguerre polynomials (see [BGX], [EMOT] :

\section{Lemma 9}

Fix $\alpha \in \mathbb{N}$. There exists $C_{\alpha}>0$ such that for $\tau \geq 0$ and $m \in \mathbb{N}$ we have:

$$
\left|L_{m}^{(\alpha)}(\tau) e^{-\frac{\tau}{2}}\right| \leq C_{\alpha}(m+1)^{\alpha}, \quad\left|\tau \frac{d}{d \tau}\left(L_{m}^{(\alpha)}(\tau) e^{-\frac{\tau}{2}}\right)\right| \leq C_{\alpha}(m+1)^{\alpha}
$$

Finally, we will exploit the following estimates, which can be easily proved by comparing the sums with the corresponding integrals: 


\section{Lemma 10}

Fix $\beta \in \mathbb{R}$. There exists $C_{\beta}>0$ such that for $0<a<b$ and $n \in \mathbb{Z}_{+}$we have:

$$
\begin{aligned}
\sum_{\substack{m \in \mathbb{N} \\
2 m+n \geq a}}(2 m+n)^{\beta} \leq C_{\beta} a^{\beta+1}, \quad \beta<-1 \\
\sum_{\substack{m \in \mathbb{N} \\
2 m+n \leq b}}(2 m+n)^{\beta} \leq C_{\beta} b^{\beta+1}, \quad \beta>-1 \\
\sum_{\substack{m \in \mathbb{N} \\
a \leq 2 m+n \leq b}}(2 m+n)^{-1} \leq \log \left(C \frac{b}{a}\right) .
\end{aligned}
$$

We can now prove the following

\section{Proposition 11}

There exists a constant $C>0$, which depends only on $n$, such that for any $\rho \in\left[N-\frac{3}{2}, N-\frac{1}{2}\right]$, $j \in \mathbb{Z}$ and $t \in \mathbb{R}^{*}$ we have:

$$
\left\|e^{-i t \sqrt{\mathcal{L}}} \psi_{j}\right\|_{L^{\infty}\left(\mathbb{H}_{n}\right)} \leq C|t|^{-\frac{1}{2}} 2^{j \rho} .
$$

Proof: Fix $t \in \mathbb{R}^{*}, j \in \mathbb{Z}$ and $(z, s) \in \mathbb{H}_{n}$. By (44), (8) and (10), putting $\sigma=\frac{s}{t}$ and $M=2 m+n$ inside the sum over $m$, we have

$$
\begin{aligned}
& e^{-i t \sqrt{\mathcal{L}}} \psi_{j}(z, s) \\
& =\frac{2^{n-1}}{\pi^{n+1}} \sum_{m=0}^{+\infty} \int_{\mathbb{R}} e^{-i t\left(\sigma \lambda+\sqrt{4 M|\lambda|+\lambda^{2}}\right)} R\left(2^{-2 j}\left(4 M|\lambda|+\lambda^{2}\right)\right) e^{-|\lambda||z|^{2}} L_{m}^{(n-1)}\left(2|\lambda||z|^{2}\right)|\lambda|^{n} d \lambda .
\end{aligned}
$$

Performing the change of variable $x=2^{-2 j} M \lambda$ we obtain

$$
e^{-i t \sqrt{\mathcal{L}}} \psi_{j}(z, s)=\frac{2^{n-1}}{\pi^{n+1}} 2^{N j} \sum_{m=0}^{+\infty} \int_{\mathbb{R}} e^{-i t 2^{2 j} g_{j, \sigma, m}(x)} h_{j, z, m}(x) d x
$$

where

$$
\begin{aligned}
g_{j, \sigma, m}(x) & =\frac{1}{M}\left(\sigma x+\sqrt{2^{2-2 j} M^{2}|x|+x^{2}}\right) \\
h_{j, z, m}(x) & =R\left(4|x|+\frac{2^{2 j} x^{2}}{M^{2}}\right) e^{-\frac{2^{2 j}|x||z|^{2}}{M}} L_{m}^{(n-1)}\left(\frac{2^{1+2 j}|x||z|^{2}}{M}\right) \frac{|x|^{n}}{M^{n+1}} .
\end{aligned}
$$

So

$$
\operatorname{supp} h_{j, z, m} \subset\left\{x \in \mathbb{R}: \frac{1}{4} \leq 4|x|+\frac{2^{2 j} x^{2}}{M^{2}} \leq 4\right\}=\left\{x \in \mathbb{R}: a_{j, m} \leq|x| \leq b_{j, m}\right\}
$$

where

$$
a_{j, m}=\frac{1}{8\left(1+\sqrt{1+2^{2 j-4} M^{-2}}\right)}, \quad b_{j, m}=\frac{2}{1+\sqrt{1+2^{2 j} M^{-2}}} .
$$


In particular

$$
b_{j, m} \leq \min \left\{1,2^{1-j} M\right\} .
$$

Note that $g_{j, \sigma, m}(-x)=g_{j,-\sigma, m}(x)$ and $h_{j, z, m}(-x)=h_{j, z, m}(x)$. Therefore, by symmetry we can consider only the integrals

$$
I_{m}=\int_{a_{m}}^{b_{m}} e^{-i t 2^{2 j} g_{m}(x)} h_{m}(x) d x
$$

where we write $g_{m}, h_{m}, a_{m}, b_{m}$ for $g_{j, \sigma, m}, h_{j, z, m}, a_{j, m}, b_{j, m}$ respectively. We prove that

$$
\sum_{m=0}^{+\infty}\left|I_{m}\right| \leq \begin{cases}C|t|^{-\frac{1}{2}} 2^{-\frac{3}{2} j}, & j \geq 0 \\ C|t|^{-\frac{1}{2}} 2^{-\frac{j}{2}}, & j<0 .\end{cases}
$$

For $x \in\left[a_{m}, b_{m}\right]$, by (23) we have

$$
\begin{aligned}
& g_{m}^{\prime}(x)=\frac{1}{M}\left(\sigma+\sqrt{1+\frac{2^{2-4 j} M^{4}}{2^{2-2 j} M^{2} x+x^{2}}}\right), \\
& g_{m}^{\prime \prime}(x)=-2^{2-4 j} M^{3}\left(2^{2-2 j} M^{2} x+x^{2}\right)^{-\frac{3}{2}}
\end{aligned}
$$

Note that by (25) we have

$$
2^{-2-2 j} M^{2} \leq 2^{2-2 j} M^{2} x+x^{2} \leq 2^{2-2 j} M^{2}, \quad x \in\left[a_{m}, b_{m}\right] .
$$

So (29) and (30) yield

$$
2^{-1-j} \leq\left|g_{m}^{\prime \prime}(x)\right| \leq 2^{5-j}, \quad x \in\left[a_{m}, b_{m}\right] .
$$

Furthermore, by Lemma 9 and (26), one can verify that

$$
\left\|h_{m}^{\prime}\right\|_{L^{1}\left(\left[a_{m}, b_{m}\right]\right)} \leq \begin{cases}C 2^{-n j} M^{n-2}, & M \leq 2^{j}, \\ C M^{-2}, & M>2^{j}\end{cases}
$$

So, by Lemma 8 with $k=2$, we obtain

$$
\left|I_{m}\right| \leq \begin{cases}C|t|^{-\frac{1}{2}} 2^{-\left(n+\frac{1}{2}\right) j} M^{n-2}, & M \leq 2^{j} \\ C|t|^{-\frac{1}{2}} 2^{-\frac{j}{2}} M^{-2}, & M>2^{j}\end{cases}
$$

For $j<0$, (27) follows directly from (33). For $n \geq 2$ and $j \geq 0$, (27) still follows from (33) by applying Lemma 10 separately to the sums $\sum_{M \leq 2^{j}}\left|I_{m}\right|$ and $\sum_{M>2^{j}}\left|I_{m}\right|$. But for $n=1$ and $j \geq 0$ this argument does not work, since we cannot apply (21) to the sum $\sum_{M \leq 2^{j}}\left|I_{m}\right|$.

So from now on we assume $n=1$ and $j \geq 0$. We divide $\mathbb{N}$ into five (possibly empty) disjoint subsets:

$$
\begin{aligned}
& A_{1}=\left\{m \in \mathbb{N}: M>2^{j}\right\}, \\
& A_{2}=\left\{m \in \mathbb{N}: M \leq 2^{j}, M \leq|t|^{-\frac{1}{2}} 2^{\frac{j}{2}}\right\}, \\
& A_{3}=\left\{m \in \mathbb{N}: M \leq 2^{j}, M>|t|^{-\frac{1}{2}} 2^{\frac{j}{2}}, \sigma \geq-\sqrt{1+2^{-1-2 j} M^{2}}\right\}, \\
& A_{4}=\left\{m \in \mathbb{N}: M \leq 2^{j}, M>|t|^{-\frac{1}{2}} 2^{\frac{j}{2}}, \sigma \leq-\sqrt{1+2^{5-2 j} M^{2}}\right\}, \\
& A_{5}=\left\{m \in \mathbb{N}: M \leq 2^{j}, M>|t|^{-\frac{1}{2}} 2^{\frac{j}{2}},-\sqrt{1+2^{5-2 j} M^{2}}<\sigma<-\sqrt{1+2^{-1-2 j} M^{2}}\right\} .
\end{aligned}
$$


Then our assertion reads:

$$
\sum_{m \in A_{r}}\left|I_{m}\right| \leq C|t|^{-\frac{1}{2}} 2^{-\frac{3}{2} j}, \quad r=1, \ldots, 5, A_{r} \neq \emptyset
$$

We prove (34) separately for each $r$, using each time Lemma 10 precisely, we will use (20) for $r=1,3,4$, (21) for $r=2$ and (22) for $r=5$. The case $r=1$ can be treated as for $n \geq 2$. For $r=2$ we estimate $\sum_{M \leq|t|^{-\frac{1}{2}} 2^{\frac{j}{2}}}\left|I_{m}\right|$ by means of the inequality

$$
\left|I_{m}\right| \leq C\left\|h_{m}\right\|_{L^{1}\left(\left[a_{m}, b_{m}\right]\right)} \leq C 2^{-2 j}
$$

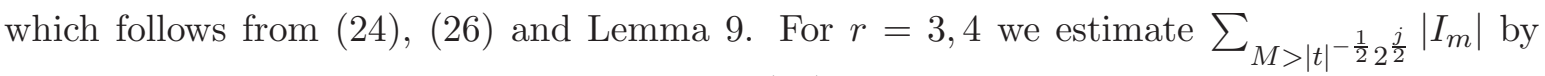
means of Lemma 8 applied with $k=1$, using (32) and the estimates

$$
\begin{aligned}
g_{m}^{\prime}(x) & \geq \frac{1}{M}\left(\sqrt{1+2^{-2 j} M^{2}}-\sqrt{1+2^{-1-2 j} M^{2}}\right) \geq C 2^{-2 j} M, \quad m \in A_{3}, \\
-g_{m}^{\prime}(x) & \geq \frac{1}{M}\left(\sqrt{1+2^{5-2 j} M^{2}}-\sqrt{1+2^{4-2 j} M^{2}}\right) \text { geqC} 2^{-2 j} M, \quad m \in A_{4},
\end{aligned}
$$

which are consequences of (28) and (30). For $r=5$ we note that $A_{5} \neq \emptyset$ implies $\sigma<-1$ and $M \in J=\left(2^{j-\frac{5}{2}} \sqrt{\sigma^{2}-1}, 2^{j+\frac{1}{2}} \sqrt{\sigma^{2}-1}\right)$. Then we estimate $\sum_{M \in J}\left|I_{m}\right|$ by means of Lemma 8 applied with $k=2$.

From Proposition [1] we can obtain, by the same proof as in BGX, pages 114-115], [FV] Corollary 10], the following

\section{Corollary 12}

For $\rho \in\left[N-\frac{3}{2}, N-\frac{1}{2}\right]$ there exists a constant $C_{\rho}>0$ such that

$$
\begin{aligned}
& \left\|e^{-i t \sqrt{\mathcal{L}}} f\right\|_{L^{\infty}\left(\mathbb{H}_{n}\right)} \leq C_{\rho}|t|^{-\frac{1}{2}}\|f\|_{\dot{B}_{1}^{\rho, 1}(\mathcal{L})}, \quad f \in \mathcal{S}\left(\mathbb{H}_{n}\right), t \in \mathbb{R}^{*}, \\
& \left\|e^{-i t \sqrt{\mathcal{L}}} f\right\|_{\dot{B}_{\infty}^{-1,1}(\mathcal{L})} \leq C_{\rho}|t|^{-\frac{1}{2}}\|f\|_{\dot{B}_{1}^{\rho-1,1}(\mathcal{L})}, \quad f \in \mathcal{S}\left(\mathbb{H}_{n}\right), t \in \mathbb{R}^{*} .
\end{aligned}
$$

The proof of the dispersive inequality is now straightforward.

Proof of Proposition [1] By (35) we obtain

$$
\left\|\cos t \sqrt{\mathcal{L}} u_{0}\right\|_{L^{\infty}\left(\mathbb{H}_{n}\right)} \leq C|t|^{-\frac{1}{2}}\left\|u_{0}\right\|_{\dot{B}_{1}^{\rho, 1}(\mathcal{L})}
$$

and by (17), (15) and (36) we obtain

$$
\begin{aligned}
\left\|\frac{\sin t \sqrt{\mathcal{L}}}{\sqrt{\mathcal{L}}} u_{1}\right\|_{L^{\infty}\left(\mathbb{H}_{n}\right)} & \leq \sum_{j \in \mathbb{Z}}\left\|\frac{\sin t \sqrt{\mathcal{L}}}{\sqrt{\mathcal{L}}} u_{1} * \psi_{j}\right\|_{L^{\infty}\left(\mathbb{H}_{n}\right)} \\
& \leq \sum_{j \in \mathbb{Z}} 2^{-j}\left\|\sin t \sqrt{\mathcal{L}} u_{1} * \psi_{j}\right\|_{L^{\infty}\left(\mathbb{H}_{n}\right)} \leq C|t|^{-\frac{1}{2}}\left\|u_{1}\right\|_{\dot{B}_{1}^{\rho-1,1}(\mathcal{L})} .
\end{aligned}
$$




\section{$5 \quad$ Strichartz inequalities}

We can now prove Theorem 2 and Corollary 3 .

\section{Proof of Theorem 2}

By (2) we can write

$$
\partial_{t} v(t)=-\frac{e^{i t \sqrt{\mathcal{L}}}-e^{-i t \sqrt{\mathcal{L}}}}{2 i} \sqrt{\mathcal{L}} u_{0}+\frac{e^{i t \sqrt{\mathcal{L}}}+e^{-i t \sqrt{\mathcal{L}}}}{2} u_{1}
$$

where $\sqrt{\mathcal{L}} u_{0}$ and $u_{1}$ both belong to $L^{2}\left(\mathbb{H}_{n}\right)$. Analogously, by (3)

$$
\partial_{t} w(t)=\int_{0}^{t} \frac{e^{i(t-\sigma) \sqrt{\mathcal{L}}}+e^{-i(t-\sigma) \sqrt{\mathcal{L}}}}{2} f(\sigma) d \sigma .
$$

So

$$
\begin{aligned}
& \|v\|_{L_{\mathbb{R}}^{p_{1}}\left(\dot{B}_{r_{1}}^{\rho_{1}, 2}(\mathcal{L})\right)}+\left\|\partial_{t} v\right\|_{L_{\mathbb{R}}^{p_{1}}\left(\dot{B}_{r_{1}}^{\rho_{1}-1,2}(\mathcal{L})\right)} \\
& \leq C\left(\|\sqrt{\mathcal{L}} v\|_{L_{\mathbb{R}}^{p_{1}}\left(\dot{B}_{r_{1}}^{\rho_{1}-1,2}(\mathcal{L})\right)}+\left\|\partial_{t} v\right\|_{L_{\mathbb{R}}^{p_{1}}\left(\dot{B}_{r_{1}}^{\rho_{1}-1,2}(\mathcal{L})\right)}\right) \\
& \leq C\left(\left\|e^{-i t \sqrt{\mathcal{L}} \sqrt{\mathcal{L}}} u_{0}\right\|_{L_{\mathbb{R}}^{p_{1}}\left(\dot{B}_{r_{1}}^{\rho_{1}-1,2}(\mathcal{L})\right)}+\left\|e^{-i t \sqrt{\mathcal{L}}} u_{1}\right\|_{L_{\mathbb{R}}^{p_{1}}\left(\dot{B}_{r_{1}}^{\rho_{1}-1,2}(\mathcal{L})\right)}\right)
\end{aligned}
$$

and

$$
\begin{aligned}
& \|w\|_{L_{I}^{p_{1}}\left(\dot{B}_{r_{1}}^{\rho_{1}, 2}(\mathcal{L})\right)}+\left\|\partial_{t} w\right\|_{L_{I}^{p_{1}}\left(\dot{B}_{r_{1}}^{\rho_{1}-1,2}(\mathcal{L})\right)} \\
& \leq C\left(\|\sqrt{\mathcal{L}} w\|_{L_{I}^{p_{1}}\left(\dot{B}_{r_{1}}^{\rho_{1}-1,2}(\mathcal{L})\right)}+\left\|\partial_{t} w\right\|_{L_{I}^{p_{1}}\left(\dot{B}_{r_{1}}^{\rho_{1}-1,2}(\mathcal{L})\right)}\right) \\
& \leq C\left(\left\|\int_{0}^{t} e^{i(t-\sigma) \sqrt{\mathcal{L}}} f(\sigma) d \sigma\right\|_{L_{I}^{p_{1}}\left(\dot{B}_{r_{1}}^{\rho_{1}-1,2}(\mathcal{L})\right)}+\left\|\int_{0}^{t} e^{-i(t-\sigma) \sqrt{\mathcal{L}}} f(\sigma) d \sigma\right\|_{L_{I}^{p_{1}}\left(\dot{B}_{r_{1}}^{\rho_{1}-1,2}(\mathcal{L})\right)}\right) .
\end{aligned}
$$

Theorem 2 follows therefore easily by the following one, where we have renamed $\rho_{1}$ the value $\rho_{1}-1$.

\section{Theorem 13}

Let $r_{1}, r_{2} \in[2, \infty]$. Let $\rho_{1}, \rho_{2} \in \mathbb{R}$ and $p_{1}, p_{2} \in[1, \infty]$ such that:

a) $\frac{2}{p_{i}}=\frac{1}{2}-\frac{1}{r_{i}}$ for $i=1,2$;

b) $-\left(N-\frac{1}{2}\right)\left(\frac{1}{2}-\frac{1}{r_{i}}\right) \leq \rho_{i} \leq-\left(N-\frac{3}{2}\right)\left(\frac{1}{2}-\frac{1}{r_{i}}\right)$ for $i=1,2$.

Let $r_{i}^{\prime}, p_{i}^{\prime}$ such that $\frac{1}{r_{i}^{\prime}}+\frac{1}{r_{i}}=1$ and $\frac{1}{p_{i}^{\prime}}+\frac{1}{p_{i}}=1$ for $i=1,2$. Then for every interval $I$ which contains 0 the following estimates are satisfied:

$$
\begin{aligned}
\left\|e^{-i t \sqrt{\mathcal{L}}} u_{0}\right\|_{L_{\mathbb{R}}^{p_{1}}\left(\dot{B}_{r_{1}}^{\rho_{1}, 2}(\mathcal{L})\right)} & \leq C\left\|u_{0}\right\|_{L^{2}\left(\mathbb{H}_{n}\right)} \\
\left\|\int_{0}^{t} e^{ \pm i(t-\sigma) \sqrt{\mathcal{L}}} f(\sigma) d \sigma\right\|_{L_{I}^{p_{1}}\left(\dot{B}_{r_{1}}^{\rho_{1}, 2}(\mathcal{L})\right)} & \leq C\|f\|_{\left.L_{I}^{p_{2}^{\prime}\left(\dot{B}_{r_{2}^{\prime}}^{\prime} \rho_{2}, 2\right.}(\mathcal{L})\right)}
\end{aligned}
$$

where the constant $C>0$ depends neither on $u_{0}, f$ nor on the interval $I$. 
We omit the proof of Theorem [13] in fact, once we have obtained Proposition [1] the procedure is classical and a good reference is given, for example, by the papers by Ginibre and Velo $(\underline{G V})$ or by Ginibre (Gi $)$. A detailed presentation in this framework is also given by [FV].

Proof of Corollary [3; Let us remark first that for $\rho_{1} \geq 0$, Proposition [ 6 ((viii) and (ix)) implies

$$
\dot{B}_{r_{1}}^{\rho_{1}, 2}(\mathcal{L}) \subset \dot{B}_{r_{\min }}^{0,2}(\mathcal{L}) \cap \dot{B}_{r_{\max }}^{0,2}(\mathcal{L}) \subset L^{r_{\min }}\left(\mathbb{H}_{n}\right) \cap L^{r_{\max }}\left(\mathbb{H}_{n}\right),
$$

where $\frac{1}{r_{\min }}=\frac{1}{r_{1}}-\frac{\rho_{1}}{N}$ and $\frac{1}{r_{\max }}=\frac{1}{r_{1}}-\frac{\rho_{1}}{N-1}$. If we take $\rho_{1}=-\left(N-\frac{3}{2}\right)\left(\frac{1}{2}-\frac{1}{r_{1}}\right)+1$ in Theorem 2, we have $\rho_{1} \geq 0$ if and only if $r_{1} \leq \frac{2(2 N-3)}{2 N-7}$. Taking into account also the condition $r_{1} \geq 2$, which corresponds to $\rho_{1} \leq 1$, we obtain by (37) the extremal spaces

$$
\begin{aligned}
u=v+w & \in L_{I}^{\infty}\left(\dot{B}_{2}^{1,2}(\mathcal{L})\right) \cap L_{I}^{2 N-3}\left(\dot{B}_{\frac{2(2 N-3)}{2 N-7}}^{0,2}(\mathcal{L})\right) \\
& \subset L_{I}^{\infty}\left(L^{\frac{2 N}{N-2}}\left(\mathbb{H}_{n}\right) \cap L^{\frac{2(N-1)}{N-3}}\left(\mathbb{H}_{n}\right)\right) \cap L_{I}^{2 N-3}\left(L^{\frac{2(2 N-3)}{2 N-7}}\left(\mathbb{H}_{n}\right)\right) .
\end{aligned}
$$

On the other hand, taking $\rho_{1}=-\left(N-\frac{1}{2}\right)\left(\frac{1}{2}-\frac{1}{r_{1}}\right)+1$ in Theorem 2] we have $\rho_{1} \geq 0$ if and only if $r_{1} \leq \frac{2(2 N-1)}{2 N-5}$. The other bound $r_{1} \geq 2$ still corresponds to $\rho_{1} \leq 1$ and we obtain therefore the extremal spaces

$$
\begin{aligned}
u=v+w & \in L_{I}^{\infty}\left(\dot{B}_{2}^{1,2}(\mathcal{L})\right) \cap L_{I}^{2 N-1}\left(\dot{B}_{\frac{2(2 N-1)}{2 N-5}}^{0,2}(\mathcal{L})\right) \\
& \subset L_{I}^{\infty}\left(L^{\frac{2 N}{N-2}}\left(\mathbb{H}_{n}\right) \cap L^{\frac{2(N-1)}{N-3}}\left(\mathbb{H}_{n}\right)\right) \cap L_{I}^{2 N-1}\left(L^{\frac{2(2 N-1)}{2 N-5}}\left(\mathbb{H}_{n}\right)\right) .
\end{aligned}
$$

By interpolation we obtain $u \in L_{I}^{p}\left(L^{r}\left(\mathbb{H}_{n}\right)\right)$ with $0 \leq \frac{2}{p} \leq \frac{1}{2}-\frac{1}{r}$ and $(N-1)\left(\frac{1}{2}-\frac{1}{r}\right)-1 \leq$ $\frac{1}{p} \leq N\left(\frac{1}{2}-\frac{1}{r}\right)-1$

\section{About the sharpness of the dispersive estimates}

We end up this paper by discussing the sharpness of the dispersive estimate obtained in Proposition 1. Let us define the functions $v_{j} \in \mathcal{S}_{\text {rad }}\left(\mathbb{H}_{n}\right), j \in \mathbb{Z}$, by

$$
\widehat{v_{j}}(m, \lambda)= \begin{cases}R\left(2^{-2 j}\left(4 n \lambda+\lambda^{2}\right)\right), & \text { if } m=0, \lambda>0, \\ 0, & \text { otherwise. }\end{cases}
$$

\section{Lemma 14}

For any $\rho \in \mathbb{R}$ there exists $C_{\rho}>0$ such that

$$
\left\|v_{j}\right\|_{\dot{B}_{1}^{\rho, 1}(\mathcal{L})} \leq C_{\rho} 2^{j \rho}, \quad j \in \mathbb{Z}
$$


Proof: We just have to prove the uniform estimate $\left\|v_{j}\right\|_{L^{1}\left(\mathbb{H}_{n}\right)} \leq C, j \in \mathbb{Z}$. Indeed $\widehat{v_{j} * \psi_{k}}(m, \lambda)= \begin{cases}R\left(2^{-2 j}\left(4 n \lambda+\lambda^{2}\right)\right) R\left(2^{-2 k}\left(4 n \lambda+\lambda^{2}\right)\right), & \text { if } m=0, \lambda>0 \\ 0, & \text { otherwise }\end{cases}$

implies $v_{j} * \psi_{k}=0$ if $|j-k| \geq 2$. Therefore by (14)

$$
\begin{aligned}
\left\|v_{j}\right\|_{\dot{B}_{1}^{\rho, 1}(\mathcal{L})} & =\sum_{k=j-1}^{j+1} 2^{k \rho}\left\|v_{j} * \psi_{k}\right\|_{L^{1}\left(\mathbb{H}_{n}\right)} \\
& \leq C_{\rho}\left\|v_{j}\right\|_{L^{1}\left(\mathbb{H}_{n}\right)} 2^{j \rho}
\end{aligned}
$$

where $C_{\rho}$ depends only on $\rho$.

Let us estimate $\left\|v_{j}\right\|_{L^{1}\left(\mathbb{H}_{n}\right)}$ :

$$
\left|v_{j}(z, s)\right|=\frac{2^{n-1}}{\pi^{n+1}}\left|\int_{\lambda_{1}}^{\lambda_{2}} e^{-i \lambda s} R\left(2^{-2 j}\left(4 n \lambda+\lambda^{2}\right)\right) e^{-\lambda|z|^{2}} \lambda^{n} d \lambda\right|,
$$

where $\lambda_{1}=\sqrt{4 n^{2}+2^{2 j-2}}-2 n$ and $\lambda_{2}=\sqrt{4 n^{2}+2^{2 j+2}}-2 n$. Then for $s \neq 0$

$$
\begin{aligned}
\left|v_{j}(z, s)\right| & =\frac{2^{n-1}}{\pi^{n+1}}\left|\int_{\lambda_{1}}^{\lambda_{2}}\left(\frac{d}{d \lambda} e^{-i \lambda s}\right) \frac{R\left(2^{-2 j}\left(4 n \lambda+\lambda^{2}\right)\right) e^{-\lambda|z|^{2}} \lambda^{n}}{i s} d \lambda\right| \\
& =\frac{2^{n-1}}{\pi^{n+1}} \frac{1}{|s|}\left|\int_{\lambda_{1}}^{\lambda_{2}} e^{-i \lambda s} \frac{d}{d \lambda}\left(R\left(2^{-2 j}\left(4 n \lambda+\lambda^{2}\right)\right) e^{-\lambda|z|^{2}} \lambda^{n}\right) d \lambda\right| \\
& =\frac{2^{n-1}}{\pi^{n+1}} \frac{1}{s^{2}}\left|\int_{\lambda_{1}}^{\lambda_{2}} e^{-i \lambda s} \frac{d^{2}}{d \lambda^{2}}\left(R\left(2^{-2 j}\left(4 n \lambda+\lambda^{2}\right)\right) e^{-\lambda|z|^{2}} \lambda^{n}\right) d \lambda\right| .
\end{aligned}
$$

So we have two possible ways to estimate $\left|v_{j}(z, s)\right|$ : using (38)

$$
\left|v_{j}(z, s)\right| \leq \begin{cases}C 2^{j(n+1)} e^{-\frac{2^{j}|z|^{2}}{C}}, & j \geq 0 \\ C 2^{2 j(n+1)} e^{-\frac{2^{2 j}|z|^{2}}{C}}, & j<0\end{cases}
$$

or using (39)

$$
\left|v_{j}(z, s)\right| \leq \begin{cases}\frac{C}{s^{2}} 2^{j(n-1)}\left(1+2^{j}|z|^{2}+2^{2 j}|z|^{4}\right) e^{-\frac{2^{j}|z|^{2}}{C}}, & j \geq 0 \\ \frac{C}{s^{2}} 2^{2 j(n-1)}\left(1+2^{2 j}|z|^{2}+2^{4 j}|z|^{4}\right) e^{-\frac{2^{2 j}|z|^{2}}{C}}, \quad j<0 .\end{cases}
$$

For $j \geq 0$, we have by (40)

$$
\begin{aligned}
\int_{\left\{|s|<2^{-j}, z \in \mathbb{C}^{n}\right\}}\left|v_{j}(z, s)\right| d z d s & \leq C 2^{j(n+1)}\left(\int_{\left\{|s|<2^{-j}\right\}} d s\right)\left(\int_{\mathbb{C}^{n}} e^{-\frac{2^{j}|z|^{2}}{C}} d z\right) \\
& =C 2^{j}\left(\int_{\left\{|s|<2^{-j}\right\}} d s\right)\left(\int_{\mathbb{C}^{n}} e^{-\frac{|w|^{2}}{C}} d w\right) \leq C
\end{aligned}
$$

and by (41)

$$
\begin{aligned}
\int_{\left\{|s| \geq 2^{-j}, z \in \mathbb{C}^{n}\right\}}\left|v_{j}(z, s)\right| d z d s & \leq C 2^{j(n-1)}\left(\int_{\left\{|s| \geq 2^{-j}\right\}} \frac{1}{s^{2}} d s\right)\left(\int_{\mathbb{C}^{n}}\left(1+2^{j}|z|^{2}+2^{2 j}|z|^{4}\right) e^{-\frac{2^{j}|z|^{2}}{C}} d z\right) \\
& =C 2^{-j}\left(\int_{\left\{|s| \geq 2^{-j}\right\}} \frac{1}{s^{2}} d s\right)\left(\int_{\mathbb{C}^{n}}\left(1+|w|^{2}+|w|^{4}\right) e^{-\frac{|w|^{2}}{C}} d w\right) \leq C .
\end{aligned}
$$


Therefore

$$
\left\|v_{j}\right\|_{L^{1}\left(\mathbb{H}_{n}\right)}=\int_{\left\{|s|<2^{-j}, z \in \mathbb{C}^{n}\right\}}\left|v_{j}(z, s)\right| d z d s+\int_{\left\{|s| \geq 2^{-j}, z \in \mathbb{C}^{n}\right\}}\left|v_{j}(z, s)\right| d z d s \leq C .
$$

Similarly, for $j<0$, we have

$$
\left\|v_{j}\right\|_{L^{1}\left(\mathbb{H}_{n}\right)}=\int_{\left\{|s|<2^{-2 j}, z \in \mathbb{C}^{n}\right\}}\left|v_{j}(z, s)\right| d z d s+\int_{\left\{|s| \geq 2^{-2 j}, z \in \mathbb{C}^{n}\right\}}\left|v_{j}(z, s)\right| d z d s \leq C .
$$

By the definition of the functions $v_{j}$ we have

$$
\cos (t \sqrt{\mathcal{L}}) v_{j}\left(0, \sigma_{j} t\right)=C 2^{N j} \int_{0}^{+\infty} e^{-i t 2^{2 j} g_{j}(x)} h_{j}(x) d x+C 2^{N j} \int_{0}^{+\infty} e^{-i t 2^{2 j} \tilde{g}_{j}(x)} h_{j}(x) d x
$$

where $\sigma_{j}$ is a constant depending only on $j, g_{j}=g_{j, \sigma_{j}, 0}$ and $h_{j}=h_{j, 0,0}$ are the functions defined in (23) and (24) respectively, and

$$
\tilde{g}_{j}(x)=\frac{1}{n}\left(\sigma_{j} x-\sqrt{2^{2-2 j} n^{2} x+x^{2}}\right) .
$$

\section{Lemma 15}

For any $j \in \mathbb{Z}$ let $\eta_{j}$ be a function in $C^{2}(\mathbb{R})$ with supp $\eta_{j} \subset\left[a_{j}, b_{j}\right]$ and let $\gamma_{j}$ be a realvalued function in $C^{4}\left(\left[a_{j}, b_{j}\right]\right)$ with $\gamma_{j}^{\prime}\left(x_{j}\right)=0$ for some $x_{j} \in\left(a_{j}, b_{j}\right)$ and $\gamma_{j}^{\prime \prime}(x) \neq 0$ for any $x \in\left[a_{j}, b_{j}\right]$. Therefore there exists $T_{j}>0$ such that

$$
\left|\int_{a_{j}}^{b_{j}} e^{-i t 2^{2 j} \gamma_{j}(x)} \eta_{j}(x) d x\right| \geq \frac{\sqrt{\pi}}{2} t^{-\frac{1}{2}} 2^{-j}\left|\gamma_{j}^{\prime \prime}\left(x_{j}\right)\right|^{-\frac{1}{2}}\left|\eta_{j}\left(x_{j}\right)\right|, \quad t>T_{j} .
$$

Proof: It is not restrictive to suppose $\gamma_{j}\left(x_{j}\right)=0$ and $\eta_{j}\left(x_{j}\right) \neq 0$. Let $\xi_{j}$ be the function defined by

$$
\xi_{j}(x)= \begin{cases}-\sqrt{\frac{2 \gamma_{j}(x)}{\gamma_{j}^{\prime \prime}\left(x_{j}\right)}}, & x \in\left[a_{j}, x_{j}\right] \\ \sqrt{\frac{2 \gamma_{j}(x)}{\gamma_{j}^{\prime \prime}\left(x_{j}\right)}}, & x \in\left(x_{j}, b_{j}\right] .\end{cases}
$$

It is not hard to check that $\xi_{j} \in C^{3}\left(\left[a_{j}, b_{j}\right]\right), \xi_{j}^{\prime}>0$ on $\left[a_{j}, b_{j}\right]$ and $\xi_{j}^{\prime}\left(x_{j}\right)=1$. Performing the change of variable $y=\xi_{j}(x)$

$$
\int_{a_{j}}^{b_{j}} e^{-i t 2^{2 j} \gamma_{j}(x)} \eta_{j}(x) d x=\int_{\xi_{j}\left(a_{j}\right)}^{\xi_{j}\left(b_{j}\right)} e^{-i t 2^{2 j} \frac{\gamma_{j}^{\prime \prime}\left(x_{j}\right)}{2} y^{2}} \Phi_{j}(y) d y,
$$

where $\Phi_{j} \in C^{2}$ and $\Phi_{j}(y)=\left(\eta_{j}\left(\xi_{j}^{-1}(y)\right)\left(\xi_{j}^{-1}\right)^{\prime}(y)\right.$, supp $\Phi_{j} \subset\left[\xi_{j}\left(a_{j}\right), \xi_{j}\left(b_{j}\right)\right]$ and $\Phi_{j}(0)=$ $\eta_{j}\left(x_{j}\right)$. We can write

$$
\int_{\xi_{j}\left(a_{j}\right)}^{\xi_{j}\left(b_{j}\right)} e^{-i t 2^{2 j} \frac{\gamma_{j}^{\prime \prime}\left(x_{j}\right)}{2} y^{2}} \Phi_{j}(y) d y=J_{j, t}+K_{j, t}
$$


where

$$
J_{j, t}=\int_{-\infty}^{+\infty} e^{-i t 2^{2 j} \frac{\gamma_{j}^{\prime \prime}\left(x_{j}\right)}{2} y^{2}} e^{-y^{2}} \Phi_{j}(0) d y=\frac{\sqrt{\pi} \eta_{j}\left(x_{j}\right)}{\sqrt{\left|1+i t 2^{2 j} \frac{\gamma_{j}^{\prime \prime}\left(x_{j}\right)}{2}\right|}} e^{-\frac{i}{2} \arctan \left(t 2^{2 j} \frac{\gamma_{j}^{\prime \prime}\left(x_{j}\right)}{2}\right)}
$$

and

$$
\begin{aligned}
K_{j, t} & =\int_{-\infty}^{+\infty} e^{-i t 2^{2 j} \frac{\gamma_{j}^{\prime \prime}\left(x_{j}\right)}{2} y^{2}}\left(\Phi_{j}(y)-e^{-y^{2}} \Phi_{j}(0)\right) d y \\
& =-\int_{-\infty}^{+\infty} \frac{d}{d y}\left(e^{-i t 2^{2 j} \frac{\gamma_{j}^{\prime \prime}\left(x_{j}\right)}{2} y^{2}}\right) \frac{\Phi_{j}(y)-e^{-y^{2}} \Phi_{j}(0)}{i t 2^{2 j} \gamma_{j}^{\prime \prime}\left(x_{j}\right) y} d y \\
& =\frac{1}{i t 2^{2 j} \gamma_{j}^{\prime \prime}\left(x_{j}\right)} \int_{-\infty}^{+\infty} e^{-i t 2^{2 j} \frac{\gamma_{j}^{\prime \prime}\left(x_{j}\right)}{2} y^{2}} \frac{d}{d y}\left(\frac{\Phi_{j}(y)-e^{-y^{2}} \Phi_{j}(0)}{y}\right) d y
\end{aligned}
$$

Therefore

$$
\left|\int_{a_{j}}^{b_{j}} e^{-i t 2^{2 j} \gamma_{j}(x)} \eta_{j}(x) d x\right| \geq\left|J_{j, t}\right|\left|1-\frac{\left|K_{j, t}\right|}{\left|J_{j, t}\right|}\right|
$$

and, since $y \mapsto \frac{\Phi_{j}(y)-e^{-y^{2}} \Phi_{j}(0)}{y}$ is a function in $C^{1}(\mathbb{R})$ whose derivative is in $L^{1}(\mathbb{R})$, as is possible to verify by direct calculation, we have

$$
\frac{\left|K_{j, t}\right|}{\left|J_{j, t}\right|} \leq \frac{\sqrt{\left|1+i t 2^{2 j} \frac{\gamma_{j}^{\prime \prime}\left(x_{j}\right)}{2}\right|}}{\sqrt{\pi}\left|\eta_{j}\left(x_{j}\right)\right|} \frac{C_{j}}{\left|t 2^{2 j} \gamma_{j}^{\prime \prime}\left(x_{j}\right)\right|} \leq C_{j}^{\prime} t^{-\frac{1}{2}} \leq \frac{1}{2}, \quad t \geq 4\left(C_{j}^{\prime}\right)^{2},
$$

where $C_{j}$ and $C_{j}^{\prime}$ are positive constants depending on $j$ but not on $t$. Thus we obtain

$$
\left|\int_{a_{j}}^{b_{j}} e^{-i t 2^{2 j} \gamma_{j}(x)} \eta_{j}(x) d x\right| \geq \frac{\sqrt{\pi}}{2} t^{-\frac{1}{2}} 2^{-j}\left|\gamma_{j}^{\prime \prime}\left(x_{j}\right)\right|^{-\frac{1}{2}}\left|\eta_{j}\left(x_{j}\right)\right|, \quad t>T_{j} .
$$

Going back to (42), for any $j \in \mathbb{Z}$ we can fix $x_{j}>0$ such that $4 x_{j}+\frac{2^{2 j} x_{j}^{2}}{n^{2}}=1$ and $\sigma_{j}<0$ such that $g_{j}^{\prime}\left(x_{j}\right)=0$. By Lemma 15] and (31) we obtain the following lower estimates for $t>T_{j}$ :

$$
\left|\int_{0}^{+\infty} e^{-i t 2^{2 j} g_{j}(x)} h_{j}(x) d x\right| \geq \begin{cases}C t^{-\frac{1}{2}} 2^{-\left(n+\frac{1}{2}\right) j}, & \text { if } j \geq 0 \\ C t^{-\frac{1}{2}} 2^{-\frac{j}{2}}, & \text { if } j<0 .\end{cases}
$$

In order to estimate the last integral in (42) we first remark that $\tilde{g}_{j}{ }^{\prime}(x)<0$ for any $x \in$ supp $h_{j} \subset\left[a_{j}, b_{j}\right]$. Performing the change of variable $y=\tilde{g}_{j}(x)$

$$
\left|\int_{a_{j}}^{b_{j}} e^{-i t 2^{2 j} \tilde{g}_{j}(x)} h_{j}(x) d x\right|=\left|\int_{\tilde{g}_{j}\left(a_{j}\right)}^{\tilde{g}_{j}\left(b_{j}\right)} e^{-i t 2^{2 j} y} H_{j}(y) d y\right|
$$

where $H_{j} \in C^{\infty}$ and $H_{j}(y)=h_{j}\left(\tilde{g}_{j}^{-1}(y)\right)\left(\tilde{g}_{j}{ }^{-1}\right)(y)$, supp $H_{j} \subset\left[\tilde{g}_{j}\left(b_{j}\right), \tilde{g}_{j}\left(a_{j}\right)\right]$. Then, for any $j \in \mathbb{Z}$ there exist $C_{j}, T_{j}^{\prime}>0$ such that

$$
\left|\int_{0}^{+\infty} e^{-i t 2^{2 j} \tilde{g}_{j}(x)} h_{j}(x) d x\right|=\left|\widehat{H}_{j}\left(t 2^{2 j}\right)\right| \leq C_{j} t^{-1}, \quad t>T_{j}^{\prime} .
$$


By (42), (44) and (45) there exists $T_{j}^{\prime \prime}>0$ such that for $t>T_{j}^{\prime \prime}$ :

$$
\left\|\cos (t \sqrt{\mathcal{L}}) v_{j}\right\|_{L^{\infty}\left(\mathbb{H}_{n}\right)} \geq \begin{cases}C t^{-\frac{1}{2}} 2^{\left(N-n-\frac{1}{2}\right) j}, & \text { if } j \geq 0 \\ C t^{-\frac{1}{2}} 2^{\left(N-\frac{1}{2}\right) j}, & \text { if } j<0\end{cases}
$$

Sharpness in t. Estimates [46] give for instance

$$
\left\|\cos t \sqrt{\mathcal{L}} v_{0}\right\|_{L^{\infty}\left(\mathbb{H}_{n}\right)} \geq C t^{-\frac{1}{2}}, \quad t>T_{0} .
$$

So the decay in $t$ in Proposition 1 cannot be improved.

Sharpness in $\rho$. Let us suppose that for some $\rho \in \mathbb{R}$ the estimate $\|\cos t \sqrt{\mathcal{L}} f\|_{L^{\infty}\left(\mathbb{H}_{n}\right)} \leq$ $C_{\rho}|t|^{-\frac{1}{2}}\|f\|_{\dot{B}_{1}^{\rho, 1}(\mathcal{L})}$ holds for any $f \in \mathcal{S}\left(\mathbb{H}_{n}\right)$. In particular, by Lemma 14, $\left\|\cos t \sqrt{\mathcal{L}} v_{j}\right\|_{L^{\infty}\left(\mathbb{H}_{n}\right)} \leq$ $C_{\rho}|t|^{-\frac{1}{2}} 2^{j \rho}, j \in \mathbb{Z}$. Estimates (46) force $\rho \in\left[N-n-\frac{1}{2}, N-\frac{1}{2}\right]$.

Final remarks. We would like to emphasise that there is no hope to obtain a dispersive inequality as in Proposition 1 with the spaces $\dot{B}_{r}^{\rho, q}(\Delta)$. Let us define the functions $w_{j} \in$ $\mathcal{S}_{\text {rad }}\left(\mathbb{H}_{n}\right), j \in \mathbb{Z}$, by

$$
\widehat{w_{j}}(m, \lambda)= \begin{cases}R\left(2^{2-2 j} n \lambda\right), & \text { if } m=0, \lambda>0 \\ 0, & \text { otherwise }\end{cases}
$$

By the inversion formula (41)

$$
\begin{aligned}
w_{j}(z, s) & =\frac{2^{n-1}}{\pi^{n+1}} \int_{0}^{+\infty} e^{-i \lambda s} R\left(2^{2-2 j} n \lambda\right) e^{-\lambda|z|^{2}} \lambda^{n} d \lambda \\
& =\frac{2^{n-1}}{\pi^{n+1}} 2^{N j} \int_{0}^{+\infty} e^{-i \nu 2^{2 j} s} R(4 n \nu) e^{-\nu\left|2^{j} z\right|^{2}} \nu^{n} d \nu \\
& =2^{N j} w_{0}\left(2^{j} z, 2^{2 j} s\right) .
\end{aligned}
$$

Therefore $\left\|w_{j}\right\|_{L^{1}\left(\mathbb{H}_{n}\right)}=\left\|w_{0}\right\|_{L^{1}\left(\mathbb{H}_{n}\right)}$. This implies, as for the functions $v_{j}$ (see the proof of Lemma 14), that $\left\|w_{j}\right\|_{\dot{B}_{1}^{\rho, 1}(\Delta)} \leq C_{\rho} 2^{j \rho}$, where $C_{\rho}$ depends only on $\rho$. By the definition of $w_{j}$ we have

$$
\cos t \sqrt{\mathcal{L}} w_{j}\left(0, \sigma_{j} t\right)=C 2^{N j} \int_{0}^{+\infty} e^{-i t 2^{2 j} g_{j}(x)} k(x) d x+C 2^{N j} \int_{0}^{+\infty} e^{-i t 2^{2 j} \tilde{g}_{j}(x)} k(x) d x
$$

where $\sigma_{j}$ is a constant depending only on $j, g_{j}=g_{j, \sigma_{j}, 0}$ and $\tilde{g}_{j}$ are the functions defined in (23) and (43) respectively, and $k(x)=R(4 x) \frac{x^{n}}{n^{n+1}}$. For any $j \in \mathbb{Z}$ we fix $x_{j}=\frac{1}{4}$ and $\sigma_{j}<0$ such that $g_{j}^{\prime}\left(\frac{1}{4}\right)=0$. Arguing as before (see the proof of [46) ) we obtain for $t>T_{j}$

$$
\left\|\cos t \sqrt{\mathcal{L}} w_{j}\right\|_{L^{\infty}\left(\mathbb{H}_{n}\right)} \geq \begin{cases}C t^{-\frac{1}{2}} 2^{(N+1) j}, & \text { if } j \geq 0 \\ C t^{-\frac{1}{2}} 2^{\left(N-\frac{1}{2}\right) j}, & \text { if } j<0\end{cases}
$$

These estimates imply that there is no $\rho \in \mathbb{R}$ for which $\|\cos t \sqrt{\mathcal{L}} f\|_{L^{\infty}\left(\mathbb{H}_{n}\right)} \leq C|t|^{-\frac{1}{2}}\|f\|_{\dot{B}_{1}^{\rho, 1}(\Delta)}$ for any $f \in \mathcal{S}\left(\mathbb{H}_{n}\right)$. 
As a conclusion we would like to remark that analysing the wave equation related to the Kohn-Laplacian $\Delta$ with the spaces $\dot{B}_{r}^{\rho, q}(\mathcal{L})$ we obtain the dispersive inequality for the wave semigroup: for any $\rho \in\left[N-\frac{3}{2}, N-\frac{1}{2}\right]$

$$
\left\|e^{-i t \sqrt{\Delta}} f\right\|_{L^{\infty}\left(\mathbb{H}_{n}\right)} \leq C_{\rho}|t|^{-\frac{1}{2}}\|f\|_{\dot{B}_{1}^{\rho, 1}(\mathcal{L})}, \quad f \in \mathcal{S}\left(\mathbb{H}_{n}\right), t \in \mathbb{R}^{*}
$$

This result does not give Proposition 1 (unless $u_{1}=0$ ) because estimate (15) does not hold with $L=\Delta$ and $\Delta_{j} u=u * \psi_{j}$.

Finally, for the Schrödinger equation related to the full Laplacian, by Proposition 7 and [FV] Corollary 10] we have the dispersive estimate

$$
\left\|e^{-i t \mathcal{L}} f\right\|_{L^{\infty}\left(\mathbb{H}_{n}\right)} \leq C t^{-\frac{1}{2}}\|f\|_{\dot{B}_{1}^{N-2,1}(\mathcal{L})}, \quad f \in \mathcal{S}\left(\mathbb{H}_{n}\right), t>0 .
$$

By a direct computation as in Section 4 the estimate (47) cannot be improved. So the behaviour of the Schrödinger operator $e^{-i t \mathcal{L}}$ by analysing it with the spaces $\dot{B}_{r}^{\rho, q}(\mathcal{L})$ is the same as in $[\mathrm{FV}]$ with the spaces $\dot{B}_{r}^{\rho, q}(\Delta)$.

\section{References}

[A] G. Alexopoulos, Spectral multipliers on Lie groups of polynomial growth. Proc. Amer. Math. Soc. 120 (1994), 973-979.

[BG] H. Bahouri and I. Gallagher, Paraproduit sur le groupe de Heisenberg et applications. Rev. Mat. Iberoamericana 17 (2001), 69-105.

[BGX] H. Bahouri, P. Gérard and C.--J. Xu, Espaces de Besov et estimations de Strichartz généralisées sur le groupe de Heisenberg. J. Anal. Math. 82 (2000), 93-118.

[BJR] C. Benson, J. Jenkins and G. Ratcliff, The spherical transform of a Schwartz function on the Heisenberg group. J. Funct. Anal. 154 (1998), 379-423.

[BJRW] C. Benson, J. Jenkins, G. Ratcliff and T. Worku, Spectra for Gelfand pairs associated with the Heisenberg group. Colloq. Math. 71 (1996), 305-328.

[C] M. Christ, $L^{p}$ bounds for spectral multipliers on nilpotent groups. Trans. Amer. Math. Soc. 328 (1991), 73-81.

[EMOT] A. Erdélyi, W. Magnus, F. Oberhettinger and F.G. Tricomi, Higher transcendental functions, Vol. 2. McGraw-Hill, New York, 1953.

[F] J. Faraut, Analyse harmonique et fonctions spéciales, in: Deux cours d'analyse harmonique. Progr. Math. 69, Birkhäuser, Boston, 1987.

[FMV] G. Furioli, C. Melzi and A. Veneruso, Littlewood-Paley decompositions and Besov spaces on Lie groups of polynomial growth. Preprint, available at http://arxiv.org/abs/math.CA/0502406 
[FV] G. Furioli and A. Veneruso, Strichartz inequalities for the Schrödinger equation with the full Laplacian on the Heisenberg group. Studia Math. 160 (2004), 157178.

[Ge] D. Geller, Fourier analysis on the Heisenberg group. Proc. Natl. Acad. Sci. USA 74 (1977), 1328-1331.

[Gi] J. Ginibre, An introduction to nonlinear Schrödinger equations, in: Nonlinear waves (Sapporo, 1995). Agemi et al. (ed.), GAKUTO Internat. Ser. Math. Sci. Appl. 10, 1997, 85-133.

[GV] J. Ginibre and G. Velo, Generalized Strichartz inequalities for the wave equation. J. Funct. Anal. 133 (1995), 50-68.

[He] W. Hebisch, Multiplier theorem on generalized Heisenberg groups. Colloq. Math. 65 (1993), 231-239.

[Hu] A. Hulanicki, A functional calculus for Rockland operators on nilpotent Lie groups. Studia Math. 78 (1984), 253-266.

[KVW] A. Korányi, S. Vági and G.V. Welland, Remarks on the Cauchy integral and the conjugate function in generalized half-planes. J. Math. Mech. 19 (1970), 10691081.

[M] G. Mauceri, Maximal operators and Riesz means on stratified groups. Sympos. Math. 29 (1987), 47-62.

[MRS1] D. Müller, F. Ricci and E.M. Stein, Marcinkiewicz multipliers and multi-parameter structure on the Heisenberg(-type) groups, I. Invent. Math. 119 (1995), 199-233.

[MRS2] D. Müller, F. Ricci and E.M. Stein, Marcinkiewicz multipliers and multi-parameter structure on the Heisenberg(-type) groups, II. Math. Z. 221 (1996), 267-291.

[MS] D. Müller and E.M. Stein, On spectral multipliers for Heisenberg and related groups. J. Math. Pures Appl. 73 (1994), 413-440.

[N] A.I. Nachman, The wave equation on the Heisenberg group. Comm. Partial Differential Equations 7 (1982), 675-714.

[P] J. Peetre, New thoughts on Besov spaces. Duke Univ. Math. Series 1, Duke University, Durham, 1976.

[S1] L. Skrzypczak, Atomic decompositions on manifolds with bounded geometry. Forum Math. 10 (1998), 19-38.

[S2] L. Skrzypczak, Besov spaces and Hausdorff dimension for some CarnotCarathéodory metric spaces. Canad. J. Math. 54 (2002), 1280-1304. 
[St] E.M. Stein, Harmonic analysis. Princeton Math. Series 43, Princeton University Press, Princeton, 1993.

[V] A. Veneruso, Schwartz kernels on the Heisenberg group. Boll. U.M.I. (8) 6-B (2003), 657-666. 